\title{
Epidemiology and causation: a realist view
}

\author{
Adrian Renton
}

\begin{abstract}
In this paper the controversy over how to decide whether associations between factors and diseases are causal is placed within a description of the public health and scientific relevance of epidemiology. It is argued that the rise in popularity of the Popperian view of science, together with a perception of the aims of epidemiology as being to identify appropriate public health interventions, have focussed this debate on unresolved questions of inferential logic, leaving largely unanalysed the notions of causation and of disease at the ontological level. A realist ontology of causation of disease and pathogenesis is constructed within the framework of "scientific materialism", and is shown to provide a coherent basis from which to decide causes and to deal with problems of confounding and interaction in epidemiological research. It is argued that a realist analysis identifies a richer role for epidemiology as an integral part of an ontologically unified medical science. It is this unified medical science as a whole rather than epidemiological observation or experiment which decides causes and, in turn, provides a key element to the foundations of rational public health decision making.
\end{abstract}

( $\mathcal{F}$ Epidemiol Community Health 1994;48:79-85)

The search for the causes of diseases and the development of approaches to disease prevention based on a knowledge of these is a major preoccupation of modern epidemiology. Mervyn Susser has recently traced the evolution of criteria for deciding whether an observed association between a factor and a disease arises from an underlying causal link. ${ }^{1} \mathrm{He}$ highlights the pragmatic orientation of epidemiologists towards the combined task of contributing to the scientific understanding of disease causation, and of developing a sound base of public health technology from which to prevent its development. Reflecting this twofold focus, Sander Greenland ${ }^{2}$ has suggested that there may be differences in the standards of data and rules of inference appropriate to furthering scientific advancement on the one hand and informing public health decision making on the other, while others see public health interventions as potentially providing quasi-experimental evidence to support or refute causal hypotheses. ${ }^{3}$

Susser describes a broad consensus over criteria for deciding causes, based around the codification by Austin Bradford $\mathrm{Hill}^{4}$ which held sway during the 1960 s and early 1970 s.
He argues that this consensus began to be unravelled during the late 1970s, with the adoption by some epidemiologists of Popper's falsificationist model of science, and the rejection of Hill's criteria as rules for induction, a process which Popper, following Hume, held to be extra-logical. Some from the Popperian camp have attempted to derive alternative falsificationist criteria for deciding causes, ${ }^{5}$ while others have rejected all such guidelines as mere conventions for establishing consensus ${ }^{6}$ which have little to do with logic or causation. Against the Popperians it has been argued that induction and verification represent a usable and pragmatic approach to deciding causes in epidemiology, ${ }^{7}$ and that it is possible, using a Bayesian approach, to assess when belief in a causal hypothesis may be deemed rational. ${ }^{2}$

In this paper I wish to suggest that while the preoccupation with Popper has proved valuable in re-emphasising the need to underpin empirical study with clearly defined hypotheses, it has also focussed epidemiological thinking about causes too exclusively on unresolved questions of logic, leaving entirely unanalysed the notions both of causation and of disease which are central to all branches of medical science. My aim is to show that even a preliminary analysis of what we mean by causation and disease can provide important clues as to how we might go about deciding causes, and defines for epidemiology a rich and vital role both within the wider endeavour of medical science and in informing public health decision making.

\section{The logic of causal inference}

Popper combined a passionate belief in the central importance of imaginative conjecture to scientific progress with David Hume's notion that induction is an extra-logical process, to arrive at his distinctive hypothetico-deductivist/falsificationist model of what science is and how it might proceed. In this model, statements about the causes of diseases are always hypothetical, unproved and, a priori, unprovable. They can, however, be falsified. Instead of trying to prove causal hypotheses, strenuous efforts are to be made both to find new falsifying evidence and to generate alternative empirically testable hypotheses which better explain the existing evidence. Where no such alternative hypotheses emerge, the causal hypothesis is corroborated, and may be considered as a rational basis for prevention.

Several objections have been raised to this model, both within and without epidemiology. Philosophers Feyerbrand, Schlesinger, O'Hare, ${ }^{8-10}$ and at least one epidemiologist, Susser, ${ }^{7}$ have maintained that despite its asser- 
tions to the contrary, induction remains at the heart of the Popperian analysis. Popper himself has suggested" that successful prediction of the behaviour of a system (and therefore falsification of hypotheses about the rules that govern it) depends on that system being "well isolated, stationary and recurrent". Heterogeneous populations of individuals acquiring diseases under the influence of diverse genetic and environmental factors can only rarely meet these conditions. Lastly, Susser has proposed ${ }^{1}$ that sociological pressure as much as force of reason may explain the attraction of Popper's analysis for epidemiologists. He suggests that the adoption of a philosophical standpoint from which all scientific statements are viewed as hypothetical serves to protect the epidemiologist from the unpleasant social, political, and commercial pressures which frequently follow the adoption of a committed position on the causes of a particular disease.

Notwithstanding these objections, Popper's model refocusses our attention on the need to scrutinise causal hypotheses with great rigour, and to pursue alternative explanations for data. ${ }^{612}$ However, the views of its proponents sometimes seem to be at variance over its implications for the respective roles of epidemiology and basic medical science in deciding causes. Diana Pettiti, ${ }^{13}$ in her amusing caricature of the Popperian and inductivist students, has highlighted the importance of the integration of insights deprived from epidemiology and the basic sciences for generating and testing causal hypotheses. Her attribution of this integrative approach to the Popperian camp seems to be undermined by Weed's ${ }^{5}$ rejection, on Popperian grounds, of those of Hill's criteria which pertain to biological mechanism, and which require that causal hypotheses be both plausible and coherent in biological terms. It will be the central theme of the remainder of this paper that achieving this crucial synthesis of basic medical science and epidemiology requires an analysis, not of logic, but of what sorts of things causes and diseases are. Such an analysis will prove neutral to disputes over the acceptability of inductive inference, and restore Hill's criteria which pertain to mechanisms of pathogenesis of central importance in deciding causes in medical research.

\section{The notion of causation}

The title of Susser's recent review "What is a cause and how do we know one?", alludes indirectly to the areas of philosophical thought which are of immediate relevance - ontology and epistemology. Ontology concerns itself with the questions about what sorts of things exist, ${ }^{14}$ and epistemology with questions about the nature, derivation, and scope of knowledge of these things. ${ }^{15}$ The association of notions of agency and causation with more general ontological and epistemological problems endures throughout the history of western philosophy. A brief examination of Hume's views on causation will take us straight to the heart of the current epidemiological controversy over causes. Consider the following extract from $A$ Treatise of Human Nature ${ }^{16}$ :

"Having thus discover'd or suppos'd the two relations of contiguity and succession to be essential to causes and effects, I am stopped short. ... Should anyone leave this instance and pretend to define a cause by saying it is something productive of another 'tis evident he would say nothing ... Can he give any definition of it that will not be the same with that of causation?"

and later

"It shall therefore be allowed for a moment that the production of one object by another in any one instance implies a power (of production). But it already having been proved that the power lies not in the sensible qualities of the cause; and there being nothing but the sensible qualities present to us; I ask why in other instances you presume that the same power still exists merely upon the appearance of these qualities. Your appeal to past experience decides nothing in the present case."

Hume roundly rejects any attempt to place causation in the realm of existence on two grounds. Firstly, we can have no direct perception of causation (powers) or indeed of anything but the "sensible qualities" of things. Secondly, we cannot infer from that which has obtained in the past what will obtain in the future. His notion of causation is thus more or less the habitual repetition of experience.

In rejecting Hume's position Susser accepts Kant's contention that causation is given a priori, and appeals to the spirit of pragmatism which has led modern science to adopt a realist ontology. ${ }^{1} \mathrm{He}$ deals first with the ontological question of what sorts of things causes are, and identifies three attributes of a cause:

(1) Association - a causal factor must occur in regular association with its effect.

(2) Time sequence - a causal factor must have existential priority over its effect.

(3) Direction - the occurrence of the effect is consequent upon (produced by) the occurrence of the cause.

For Susser establishing direction is the "crux of the difficulties in making a valid causal inference". While he has probably gone further than any other epidemiologist in confronting the ontological aspects of causation, at the end he sidesteps the issue of what direction is or how it may be known. Consequently, he fails to give sufficient emphasis to knowledge of the process through which causes produce their effects. It will be shown below that Susser's three properties of causation can be derived from a more fundamental analysis of the ontology of causation.

\section{The ontology of causation}

In his realist account, Mario Bunge has identified causation as a particular category of determination. Determination is taken to mean a "way of becoming" which is characterised by the principles of lawfulness and productivity. ${ }^{17}$ By lawfulness he means that "events take place (are determined) in accordance with a set of objective laws, whether we know the laws or not". By productivity he means the "ancient principle according to which nothing comes out of nothing or passes into nothing". 
According to this view causation represents a way of becoming, and requires that productivity is a real characteristic of real things.

It is necessary to expand a little on what is meant by a law in the above. Realist theories of science have tended to distinguish between laws and law statements. Bunge uses the term laws, to refer to the regular patterns of being and becoming which are taken to be objectively real. $\mathrm{Laws}_{2}$ refer to our conceptual reconstructions of laws, or verbal statements of these. Roy Bhaskar has drawn the same distinction with his notions of the intransitive and transitive objects of scientific knowledge. In this paper I shall use the word "laws" to mean laws.

If we accept Bunge's view of the real nature of causation, then Susser's "properties of causes" follow directly. A cause will be observed to be associated with its effect where observation is adequate in resolution and duration, since the production is regular in its accordance with laws. A cause is unable to produce its effect before it exists itself and is therefore, by definition, existentially prior to it. The productive nature of the relation secures direction or asymmetry.

The preceding discussion has led us to the view that causation describes a way of becoming, characterised by its regularity, and productivity. We must next examine the ontological assumptions of our notions of disease and pathogenesis.

\section{The ontology of disease and pathogenesis}

Hippocratic/Galenic medicine believed that diseases were imbalances of "humours", ${ }^{18}$ specific to individuals, and were manifest in the realm of the observable only by the clinical features. The notion that diseases were specific entities with real physical existence was developed much later by Sydenham and Baglivi in the 17th century, ${ }^{19}$ and formalised by the Paris pathologists ${ }^{20}$ in the 18 th century, thus providing the foundation for our modern ontology of disease. The profound reductionism and materialism of this ontology is witnessed by the introduction to the Muir's standard textbook of pathology for medical students. ${ }^{21}$

"Disease may be defined as any abnormal variation in structure or function of any part of the body ... disease does not occur spontaneously, but only as the consequence of some abnormality induced in the cells of the body by specific causal factors."

This quotation defines an ontology of disease (and hints at an ontology of pathogenesis) in terms of the body's material elements and its material processes. This ontology has been termed "scientific materialism" by Alfred North Whitehead ${ }^{22}$ and has dominated both the biological and physical sciences since the abandonment of vitalism. According to this view, the organ, tissues, and cellular systems of the body, in common with all other things, are composed of an "irreducible brute matter or material, spread throughout space in a flux of configurations". ${ }^{22}$ The physiological processes and mechanisms which comprise the behaviour of body systems over time are similarly determined by the laws governing the behaviour of brute matter. Thus, pathogenesis is taken to be nothing more than the interaction between physiological processes/mechanisms and any influences which may impinge upon them; and diseases represent the end stages of these processes.

Scientific materialism thus provides a unifying ontological framework within which can be integrated not only the mechanisms governing living and dead matter, but also those governing what Susser ${ }^{23}$ has called the different systems and levels of organisation of living things. Although scientific materialism is consistent, well worked out, and widely accepted, I do not believe it is the only ontological framework that might plausibly achieve such an integration. As Joseph Needham has suggested ${ }^{24}$ :

"Mechanists do not say that nothing is true or intelligible unless expressed in physico chemical terms . . . what they do say is that the processes of living matter are subject to the same laws that govern processes in dead matter."

It seems possible that other ontologies may, in the future, take hold of the scientific imagination, and in time supercede scientific materialism as the dominant influence. Candidates might include the consistent organic mechanism elaborated by Whitehead and his followers, ${ }^{25}$ which places the organism rather than brute matter at the foundation of real existence. A discussion of the relative merits of different ontologies is, however, beyond the scope of this paper. Therefore, having identified scientific materialism as the ontology which currently dominates our notions of disease and pathogenesis, I will now try to outline a realist principle of disease causation within this ontology, and to draw out its implications for epidemiology. My argument will, nonetheless, be seen to hold in its general form whatever monistic ontology we might adopt, providing that it is realist in attitude.

\section{A causal principle for epidemiology}

Having analysed the ontological content of the notions of causation, disease, and pathogenesis, we are in a position to formulate a consistent description of what we understand by a cause of a disease? Let us first consider the definitions of two of the most thoughtful writers on the subject. Mervyn Susser ${ }^{1}$ (p 637) has recently suggested the following:

"A determinant is any factor which affects an outcome (disease)."

A similar definition has been propounded by Kenneth Rothman ${ }^{26}$ as:

"An event, condition or characteristic which plays an essential role in producing an occurrence of the disease."

Both these definitions are open to the charge of circularity levelled by Hume in the first of the quotations given above. ${ }^{16}$ Susser defines a determinant as that which affects an outcome; which is just another way of saying that it 
determines it. Similarly, Rothman defines a cause as that which produces the disease, where clearly the notion of production entails the notion of causation. Does our ontological analysis offer us any way out of this trap? I have outlined above the generally accepted scientific materialist ontological model for pathogenesis. Starting from this model I would suggest:

A cause of a disease is any factor in whose presence the general laws according to which a biological process or mechanism evolves over time determine that its evolution will be towards the abnormal state which the disease represents.

It will be immediately apparent that this formulation suffers problems of circularity similar to those previously considered. However, there is an important difference. Where previously the affecting or producing was attributed to the cause itself, in the latter statement it is attributed to laws with a higher degree of generality. This point is of considerable importance. In effect, it places the status of the association between factor and disease, not at the level of an induction from repeated observations, but as a deducible particular instance of more general laws of nature. It is notable that this encompassing of particular hypotheses within more general ones is held by Popper to be a fundamental characteristic of a progressive science. ${ }^{27}$

It is clear that our formulation, as it stands, allows as causes only factors which are directly involved in biological mechanisms. According to the usual epidemiological model, the interaction between elements of the causal agent, the host, and the environment sets up a process whose final common pathway represents the disease state. A restriction of the notion of cause to factors involved in this final pathway might be deemed to place an arbitrary and unnecessary constraint upon both the explanatory power of epidemiology and its ability to contribute to the rational design of public health interventions. It is precisely here that the adoption of an ontological position becomes important. The agent, host, and environment are posited as three separate domains within the model, precisely because at the empirical level they represent different systems and levels of organisation. However, in order that they should interact they must have a common ontological status. Viewed from the scientific materialist standpoint, this status is that they comprise more or less complex systems of material processes. The elaboration of causal chains and networks constructed within this ontology is the business of all those areas of medical science which are concerned with elucidating the causation of disease.

\section{Epistemological consequences of a realist view of causation}

A realist scientific ontology requires not only that the things which are the objects of science exist and that they share the same sort of existence, but also that the laws governing change also exist in the sense of being immanent in the things themselves. It is by virtue of this realist ontology that we can integrate different phenomenal levels of organisation. Because both the individual and his gut partake of the same kind of existence we can explain his report of dyspepsia and epigastric pain by describing the ulceration of his stomach. Similarly, the assumption that both populations and individuals partake of the same kind of existence underlies the epidemiologist's conviction that the study of populations can tell us something about the determination of disease in an individual. In what follows, the adoption of a realist ontology will be seen to have particular consequences for the work of the epidemiologist.

\section{A REALIST VIEW INTEGRATES EPIDEMIOLOGY \\ WITH BIOLOGICAL SCIENCE}

The adoption of the scientific materialist ontology permits the fruitful integration of epidemiology and basic medical science. It allows us both to interpret associations between exposures and diseases observed in populations as providing clues to how diseases are determined in the individual and to use our knowledge of pathogenesis to generate hypotheses that may be tested at the epidemiological level. An example will serve to illustrate.

Last century, Rigoni Stern noticed in an analysis of statistics from Verona that death from uterine cancer was more common in married than in unmarried women. His suggestion that this might be related to sexual or reproductive activity was largely forgotten until the demonstration in the 1950s of the rarity of the disease among Catholic nuns. Casecontrol studies carried out during the 1960s which found increased risk to be associated with parameters of sexual behaviour ${ }^{28}$ were widely interpreted as suggesting a causal role for a sexually transmitted organism. ${ }^{29}$ The search for such an organism led to the association of a variety of genitourinary tract pathogens with cervix cancer. Most of these agents were later shown to be associated through confounding with number of sexual partners. ${ }^{30}$ While several case-control studies in the 1970s reported an association with herpes simplex virus II antibodies (HSV II) which occurred independently of sexual history, examination of biopsy specimens found no expression of HSV2 DNA in cervix cancer cells. Furthermore follow up studies failed to detect any excess risk of cervical intraepithelial neoplasia (CIN) in HSV infected women. ${ }^{30}$

The discovery of the oncogenic potential of animal papillomaviruses ${ }^{31}$ together with reports of malignant degeneration in human warts led to interest in the idea that human papillomavirus (HPV) might be the culprit. Evidence from epidemiological studies showing an association between genital warts and $\mathrm{CIN}^{32}$ spurred the development of new DNA hybridisation techniques whose use in recent epidemiological studies has shown that the association of HPV with cancer of the cervix is confined to particular genetic subtypes of the 
virus. Pathological studies have consistently demonstrated DNA from these subtypes in cervical cancer cells taken from a high proportion of cases, ${ }^{33}$ often integrated into the genome of these cells. ${ }^{34}$ Various HPV gene products have been shown to transform cells in model systems by binding to host cell antioncogenes. ${ }^{3536}$ Testing for oncogenic HPV types is currently being considered as an adjunct to PAP smear in cervical cancer screening programmes.

It is very difficult to see how the current widely held hypothesis that certain genetic types of HPV cause cancer of the cervix could have been arrived at without the constant cross-fertilisation between epidemiological and biological approaches to the disease. The example is by no means unique, but rather has been a general feature of the elucidation of the determinants of disease in human populations, and Susser has provided many examples in his book. ${ }^{24}$

\section{THE REALIST VIEW ASSISTS IN THE} IDENTIFICATION OF POTENTIAL CONFOUNDERS When observational studies report that a putative causal factor is associated with a disease, the possibility that the association has arisen through confounding must be considered. Attempts to exclude the effects of confounding factors from the associations described by observational studies require their measurement and control, either through matched designs, or the stratified analysis of study results. ${ }^{3839} \mathrm{~A}$ central problem with this strategy is that to measure a potential confounder in the first place, the investigator must know in advance that, potentially, it is independently associated with occurrence of the disease. But how can she have this prior knowledge?

Broadly applicable demographic factors such as age and social class are almost universally considered as potential confounders. Both of these factors are associated with marked variation in the incidence of many diseases. In the case of age this reflects both the fact that older age allows chronicity of harmful exposures and an appeal to the notion, whether or not cast in Darwinian terms, that biological systems "wear out". In the case of social class this confounding is generally explained by the clustering of harmful exposures towards one or other end of the class scale. Such implicit assumptions are pointers to the three principal ways in which the more specific potential confounders are identified for the purposes of design of observational studies.

Firstly, factors known through previous epidemiological investigation to be associated with the disease represent obvious candidates. Secondly, factors known to be associated with the putative cause may be deemed worthy of measurement. Thirdly, factors which are known to, or which theory suggests may, impinge on the mechanisms involved in pathogenesis, should be considered as potential confounders. Furthermore, where factors previously associated with the disease or the putative cause are also thought to impinge on pathogenesis, then their candidacy as confounders is strengthened.

In the previous section I have highlighted the constant cross-fertilisation between epidemiological and pathological approaches in explaining the causes of disease. In this section it has become clear that a similar process is also crucial to the control of confounding factors, because an understanding of the mechanisms of pathogenesis is of central importance to their successful identification. Nonetheless, in deciding causation from associations found in the results of observational studies we can never be sure that we have not failed adequately to control for an important confounder. This has led some epidemiologists to the view that only experimental studies in which there is an intervention to change "exposure" to a factor should claim to address the question of whether that factor is causal. ${ }^{40}$ There are two problems with this view. Firstly, the ability of such studies to isolate experimentally induced variation in the exposure from concomitant variation in any confounders will entirely depend on the origin of the putative cause/confounder association. ${ }^{1}$ Secondly, in many intervention studies, adequate definition of change in exposure in both intervention and control groups may be difficult to achieve.

THE REALIST VIEW ALLOWS A COHERENT ANALYSIS OF CAUSAL INTERACTION IN EPIDEMIOLOGICAL STUDIES

It has long been acknowledged that factors which play a role in the pathogenesis of a disease are often not in themselves necessary for the development of disease, nor in most cases sufficient. ${ }^{1}$ This understanding has been implicit in the construction of the commonly used "effect measures" which express the strength of associations between factors and diseases, and also in attempts to refine these measures to serve the analysis of the effects of multiple causation in the results of observational studies: the analysis of interaction. Cooping $^{41}$ has suggested that interest in the problem of interaction has grown with a shift in the focus of epidemiology from implicating factors in the causation of diseases towards assessment of their contribution to the overall disease problem. Discussion has turned around ways of deriving an expression for the relative risk for disease associated with exposure to one or more particular factors jointly, in terms of the relative risks associated with those factors individually, where they act through the same pathogenetic pathway to cause the disease.

In debating this problem most authors have agreed with Rothman's contention ${ }^{25}$ that the analysis of interaction between causal factors, if it is not to be entirely arbitrary, must be founded upon the notion that this interaction occurs through their involvement in the same pathogenetic mechanism. To further his analysis Rothman has developed what has been termed the component discrete causes model. ${ }^{42}$ According to this model a factor will 
cause a disease when present in combination with other factors. Such a combination represents a sufficient cause of the disease, though it may not be necessary in that there may be other combinations of factors that are equally sufficient. This model represents an abstraction from the underlying pathogenetic mechanism by removing the dimension of time; it is not maintained that all the component factors must be present together at any particular moment, but that they are present at such times as enable them to participate in the same pathogenetic mechanism. This model offers a natural definition for interaction between two factors as being their coparticipation in the same causal mechanism.

In his elaboration of the implications of this model for assessing interaction in observational data, Rothman appeared to some ${ }^{4243}$ to assert that departure from an additive model for the combination of relative risks was the only biologically plausible evidence for interaction. Proponents of the mathematically more tractable multiplicative models objected to this assertion, demonstrating that factors might coparticipate in plausible pathogenetic mechanisms in such a way as to display multiplicative interactions. ${ }^{4243} \mathrm{~A}$ broad consensus was eventually reached that interaction between factors within pathogenetic mechanisms could indeed lead to multiplicative relationships between their associated relative risks, ${ }^{44}$ but that for the purposes of public health decision making an additive criterion for the independence of effects was appropriate. ${ }^{45}$ This is not, however, to abandon the mechanistic underpinnings of the public health view. To use Rothman's own words ${ }^{25}$ :

"The Public Health consequences of interaction between factors are the manifestations of the biologic definition of interaction between component causes of disease applied to a population."

Rothman has shown that the notion of a pathogenetic mechanism provides the foundation of the analysis of interaction between factors in data derived from observational studies. But this is not all. Analysis of the arithmetic form of the relationship between the relative risks associated with different combinations of interacting factors may provide clues as to the biological nature of the interaction, ${ }^{4143}$ because, as Rothman has pointed out, multiplicative models will correspond in general to causal factors acting at different stages, and additive models to action at the same stage in a multistage process. ${ }^{44}$

\section{Deciding causes in epidemiology}

In the preceding discussion I have suggested that while the adoption of Popper's model by epidemiologists has had many positive consequences, it has led to an overemphasis on the problems of inferential logic at the expense of an analysis of what we understand by diseases and causes. Although the proponents of this model have made strenuous efforts to demarcate science and policy, their concern with the epistemological problem of inferring true statements about the causes of diseases from the results of epidemiological studies has emphasised a role for epidemiology in directing public health decision making which it cannot sustain alone. I have proposed a complementary view based on an analysis of the ontology of disease and causation in which epidemiology is characterised as a basic medical science, standing integrated with other basic medical sciences and progressing through a rich process of cross-fertilisation with these other disciplines. In this view medical science as a whole is brought to bear on the problem of deciding causes, and provides a key element to the foundations of rational public health decision making as it relates to disease prevention.

The ontology of scientific materialism, which is implicit in our modern notions of both disease and causation, offers us one framework within which we can hope to untangle the complex web of events occurring at the levels of the agent, host, and environment which determine the development of diseases. Emphasis on the real basis of disease causation serves us in a number of important ways. It helps us to identify research questions which may be addressed by epidemiological observation and allows that epidemiological observation suggest pathogenetic mechanisms which might be investigated by basic medical scientists. It provides us with a rational basis from which to identify potential confounding and effect modifying factors for which to control in epidemiological studies. It requires that statements about the causation of diseases be cast in terms of physical laws and scientific hypotheses of more general application in the physical world.

The consistent association between a factor and a disease occurring in correct time order in observational studies, where bias has been minimised, suggests a causal or confounded relationship. A strong relationship which persists in the face of strenuous attempts to control confounding in observational studies and through intervention studies shifts the balance towards causation. A knowledge of the mechanisms of pathogenesis of the disease, and the demonstration that a factor will materially influence these mechanisms through the material laws which govern them, adds further to our confidence in causation. Hill's criteria of biological gradient, plausibility, and coherence shift the epidemiologist's attention towards the real material basis of disease causation. Where there is evidence, either from basic medical science or epidemiology of causation, policy makers will consider whether the use of public health technology to modify the distribution of the factor or to identify those exposed might be possible and appropriate. Where there is both of these, properly designed public health programmes might certainly be expected to yield some success, and controlled trials, where ethi$\mathrm{cal}$, are likely to be the best way to assess their effectiveness.

1 Susser $M$. What is a cause and how do we know one? A grammar for pragmatic epidemiology. Am $\mathcal{F}$ Epidemiol 1991;7:635-48. 
2 Greenland S. Probability versus Popper: an elaboration of the insufficiency of current Popperian approaches for epidemiologic analysis. In: Rothman KJ, ed. Causal inference. Chestnut Hill, MA: Epidemiology Resources, 1988:95-104.

3 Jacobsen M. Inference in epidemiology. In: Rothman $\mathrm{KJ}$, ed. Causal inference. Chestnut Hill, MA: Epidemiology Resources, 1988:105-18.

4 Hill AB. Environment and disease: association or causation? Proc $R$ Soc Med 1965;58:295-300.

5 Weed DL. Causal criteria and Popperian refutation. In: Rothman KJ, ed. Causal inference. Chestnut Hill, MA: Rothman KJ, ed. Causal inference. Che

6 Lanes SF. The logic of causal inference. In: Rothman KJ, ed. Causal inference. Chestnut Hill, MA: Epidemiology

7 Susser M. Falsification, verification and causal inference in epidemiology: reconsideration in the light of the philosophy of Sir Karl Popper. In: Rothman KJ, ed. Causal inference. Chestnut Hill, MA: Epidemiology Resources, 1988:33-58. 8 Feyerabend P. Farewell to reason. New York: Verso,

9 Schlessinger GN. There's fascination frantic in philosophical fancies. In: Rothman KJ, ed Causal inference. Chestnut Hill, MA: Epidemiology Resources, 1988:165-72.

10 O'Hear A. Karl Popper. London: Routledge and Keegan Paul, 1980.

11 Popper K. Conjectures and refutations. London: Routledge and Keegan Paul, 1974:339.

12 McIntyre N. The truth, the whole truth and nothing but the truth. In: Rothman KJ, ed. Causal inference. Chestnut the truth. In: Rothman KJ, ed. Causal inference. Ches

13 Petitti DB. The implication of alternative views about causal inference for the work of the practising epidemiologist. In: Rothman KJ, ed. Causal inference. Chestnut Hill, MA: Epidemiology Resources, 1988:149-52.

14 Quine WVO. From a logical point of view. Cambridge, MA Harvard University Press, 1980:1-19.

15 In: Flew A, ed. A dictionary of philosophy. London: Macmillan, 1984

16 Hume D. A treatise of human nature. London: Penguin 1984:124 and 139 .

17 Bunge M. Causality and modern science. 3rd rev ed. New York: Dover Publications Inc, 1979:11.

18 Galen's hygiene. Translated by Green, RM. Springfield, Illinois: Charles C Thomas, 1951

19 Coulter HL. Divided legacy; a history of the schism in medical thought. Vol II. Washington, DC: Wehawken Book Co, 1977:180-221.

20 Foucault $M$. The birth of the clinic: an archeology of medical perception. London: Tavistock, 1973.

21 Cappell DF, Anderson JR. Muir's textbook of pathology. London: Edward Arnold Ltd, 1971:ix.

22 Whitehead AN. Science and the modern world. London: Free Association Books, 1985:22.

23 Susser M. Causal thinking in the health sciences. New York Oxford, 1973.
24 Needham J. A biologist's view of Whitehead. In: Schlipp PA, ed. The philosophy of Alfred North Whitehead. Evan ston: Northwestern University, 1941:247.

25 Whitehead AN. Process and reality. New York: The Free

26 Rothman KJ. Modern epidemiology. Boston: Little Brown and Company, 1986:11.

27 Popper KR. The logic of scientific discovery. New York: Harper and Row, 1968:276-81.

28 Rotkin I. A comparison of key epidemiological studies in cervical cancer related to current searches for transmissible agents. Cancer Res 1973;33:1353-67.

29 Buckley J, Harris R, Doll R, et al. Case control study of the husbands of women with dysplasia or carcinoma of the cervix uteri. Lancet 1981 ;ii:1011-14.

30 Oriel J. Sex and cervical cancer. Genitourin Med 1988;64:81-9.

31 Jarrett $W$. Papillomaviruses and cancer. In: Anthony $P$ MacSween R, eds. Recent advances in histopathology. Vo 11. Edinburgh: Churchill Livingstone, 1981:35-48.

32 Franchesi S, Doll R, Gallwey J, et al. Genital warts and cervical neoplasia: an epidemiological study. Br $\mathcal{Y}$ Cancer 1983;48:621-8.

33 Koutsky L, Galloway D, Holmes K. Epidemiology of genital human papillomavirus infection. Epidemiol Rev 1988;10:122-63.

34 Doeberitz M, Oltersdorf T, Schwarz E, et al. Correlation of modified human papillomavirus early gene expression with altered growth properties in C4-1 cervical carcinom with altered growth properties in C4-

35 Vousden KH: Human papillomavirus oncoproteins. Seminars in Cancer Biology 1990;1:415-24.

36 Scheffrer M, Munger K, Byrne JC, et al. The state of the $\mathrm{P} 53$ and retinoblastoma genes in human cervical carcinoma cell lines. Proc Natl Acad Sci USA 1991;88:5523-7.

37 Zur Hausen H. Papillomavirus in anogenital cancer: the dilemma of epidemiological approaches $7 N C I$ 1989;81:1680-1.

38 Breslow NE, Day NE. Statistical methods in cancer research. Vol II - The design and analysis of cohort studies. IARC Scientific Publications No 82. Lyon: IARC, 1982.

39 Schlesselman JJ. Case control studies: design, conduct, analysis. New York: Oxford University Press, 1982.

40 Petitti DB. Associations are not effects. Am $\mathcal{f}$ Epidemio 1991;133:101-2.

41 Koopman JS. Causal models and sources of interaction. $\mathrm{Am}$ f Epidemiol 1977;106:439-44.

42 Koopman JS. Interaction between discrete causes. $\mathrm{Am} \mathcal{F}$ Epidemiol 1981;113:716-24.

43 Walter SD, Holford TR. Additive, multiplicative, and other models for disease risks. Am $\mathcal{f}$ Epidemiol other models

44 Rothman KJ, Greenland S, Walker AM. Concepts of interaction. Am $\mathcal{F}$ Epidemiol 1980;112:467-70.

45 Blot WJ, Day NE. Synergism and interaction: are they equivalent. Am $\mathcal{F}$ Epidemiol 1979;110:99-100. 